\title{
The Role of Ambipolar Potential in the Propulsive Performance of the GDM Thruster
}

\author{
T. Kammash* \\ R. Tang** \\ University of Michigan \\ Ann Arbor, MI 48109
}

\begin{abstract}
$\underline{\text { Abstract }}$
Many of the studies assessing the capability of the Gasdynamic Mirror (GDM) fusion propulsion system used analyses that ignored the ambipolar potential. The electrostatic potential arises as a result of the fast escape of the electrons due to their small mass. As they escape they leave behind an excess of positive charge which manifests itself as a positive electric potential that slows down the electron escape while speeding up the ions until their respective axial diffusions are equalized. The indirect effect on the ions is that their confinement time is reduced, and to compensate for that, the length must increase, relative to that of zero potential, in order to allow for recovery of an equal amount of fusion power. But as they emerge from the thruster mirror, the ions acquire an added energy equal to the potential, and that manifests itself in increased specific impulse and thrust. We examine in this paper the underlying theory of this effect and evaluate its impact on the GDM propulsion capability.
\end{abstract}

Nomenclature

$\begin{aligned} A_{c} & =\text { area of plasma core } \\ A_{0} & =\text { mirror area } \\ D & =\text { axial diffusion coefficient } \\ E & =\text { electric field } \\ E_{e} & =\text { electron energy } \\ E_{L} & =\text { escape energy } \\ e & =\text { electron charge } \\ k & =\text { density scale length } \\ L & =\text { length of plasma }\end{aligned}$

* AIAA Associate Fellow

** Graduate student in Aerospace Engineering

$$
\begin{aligned}
\ln \Lambda & =\text { Coulomb logarithm } \\
m & =\text { particle mass } \\
n & =\text { particle density } \\
R & =\text { plasma mirror ratio } \\
T & =\text { temperature } \\
v & =\text { monoenergetic particle velocity } \\
Z & =\text { charge number } \\
\Gamma & =\text { velocity-averaged particle flux } \\
\mu & =\text { mobility } \\
\tau & =\text { confinement time } \\
\varphi & =\text { electrostatic potential } \\
v & =\text { collision frequency } \\
\tau_{R T} & =\text { Earth-Mars round trip time } \\
d & =\text { distance between Earth and Mars } \\
g & =\text { Earth's gravitational acceleration } \\
I_{s p} & =\text { specific impulse } \\
m_{f} & =\text { vehicle dry mass } \\
F & =\text { thrust }
\end{aligned}
$$

\section{Introduction}

The gasdynamic mirror (GDM) fusion propulsion system is a magnetic mirror confinement system in which a hot dense plasma is confined long enough to allow fusion reactions to take place while allowing a fraction of the charged particles to escape to produce the desired thrust ${ }^{(1)}$. It could also be used as a plasma thruster if means other than fusion power are employed to heat the plasma, e.g. radiative heating. In the latter case, the magnetic mirror confines the plasma during the heating process, after which it is ejected from the mirror end which serves as a magnetic nozzle. In both instances sufficiently long confinement is needed, and in both cases the rapid loss of the electrons gives rise to the electrostatic potential which impacts the propulsive 
performance of the device. In this paper we address the physics of such a potential and assess its effect on the escape energies of both the electrons and ions irrespective of whether the GDM is fusion-powered or heated by other means.

Mathematical Formulation

We begin with the monoenergetic diffusion equations for the electrons and ions in the device ${ }^{(2)}$ :

$$
\begin{aligned}
& (1 / R) \gamma_{e}=-D_{e} \nabla n_{e}-\mu_{e} \vec{E} n_{e} \\
& (1 / R) \gamma_{i}=-D_{i} \nabla n_{i}+\mu_{i} \vec{E} n_{i}
\end{aligned}
$$

where $R$ is the mirror ratio reflecting the fact that the monoenergetic flux $\gamma$ is measured at the throat of the mirror, where the area is $A_{0}$. If the plasma area at the center of the device is $A_{c}$, then $A_{0}=A_{c} / R$. It is assumed that the ion and electron densities vary as

$$
n=n_{c} \exp [-(2 k / L) z]
$$

where $L$ is the axial length of the system and $k$ is an integer. It is clear that we can write for the total monoenergetic flux through the mirror the result

$$
A_{0} \gamma=-A_{0} D \nabla n=(2 k / L) A_{0} D n
$$

For the ions we employ the diffusion coefficient given by

$$
D_{i}=L^{2} / 4 k \tau_{i}
$$

with

$$
\tau_{i}=R L / v_{i}
$$

and $v_{i}$ denoting the velocity of the monoenergetic ions. For the electrons we use the following diffusion coefficient:

$$
D_{e}=v_{e}^{2} / 3 v_{e i}
$$

and employ the following definitions for the mobilities given in Eq. (1), namely

$$
\begin{aligned}
& \mu_{e}=e / m_{e} v_{e i} \\
& \mu_{i}=z e / m_{i} v_{e i}
\end{aligned}
$$

In the above equations $v_{e i}$ denotes the electron-ion collision frequency represented by

$$
v_{e i}=\frac{n_{e} \ln \Lambda}{C_{0} E_{0}^{3 / 2}}
$$

where the constant $C_{0}$ is given by

$$
C_{0}=8.176 \times 10^{9} \mathrm{~s} \mathrm{~cm}^{-3} \mathrm{keV}^{-3 / 2}
$$

It is further assumed that the electrostatic potential varies in space in the same manner as the electron and ion densities so that the electric field becomes

$$
\vec{E}=-\nabla \varphi=(2 k / L) \varphi
$$

Because the plasma in GDM is highly collisional, it is reasonable to assume that the species have Maxwell-Boltzmann velocity distributions with which the total electron and ion fluxes $\Gamma_{\mathrm{e}}$ and $\Gamma_{\mathrm{i}}$ can be found ${ }^{(3)}$. The condition of charge neutrality requires that these charged fluxes be equal and the net charge be zero. Upon satisfying these conditions the following expression is obtained for the electrostatic potential $\varphi$ :

$$
\begin{aligned}
& \left(1-\frac{e \varphi}{T_{e}}\right)\left[1-e r f\left(\sqrt{\frac{3 e \varphi}{2 T_{e}}}\right)\right]+\frac{2}{\sqrt{\pi}} \sqrt{\frac{3 e \varphi}{2 T_{e}}} \exp \left(-\frac{3 e \varphi}{2 T_{e}}\right) \\
& =\frac{m_{e} L v_{e i}}{4 R k T_{e}}\left(\frac{8 T_{i}}{\pi m_{i}}\right)^{1 / 2}+\frac{m_{e}}{m_{i}} z_{i}^{2} \frac{e \varphi}{T_{e}}
\end{aligned}
$$


where $T_{e}$ and $T_{i}$ are the electron and ion temperatures respectively and

$$
\operatorname{erf}(x)=\frac{2}{\sqrt{\pi}} \int_{0}^{x} e^{-t^{2}} d t
$$

is the familiar error function. We note in Eq. (12) that the mirror ratio $R$ appears only in the ion dynamics term since only the ions respond to mirror confinement as noted earlier. Moreover, $T_{e} \neq T_{i}$ in GDM since the electron distribution is in effect a "truncated" Maxwellian because electrons with energies above that of the potential do escape. The potential is obtained from Eq. (12) when solved iteratively, and it can be used to evaluate the electron and ion escape energies, which can be expressed by

$$
E_{L e}=\left\{\frac{\left(5-2 x^{2}\right)[1-e r f(x)]+\frac{2}{\sqrt{\pi}}\left(5+\frac{4}{3} x^{2}\right) x e^{-x^{2}}}{2\left(1-\frac{2}{3} x^{2}\right)[1-e r f(x)]+\frac{4}{\sqrt{\pi}} x e^{-x^{2}}}\right\} T_{e}
$$

$$
E_{L i}=\left[\frac{2+\frac{m_{e}}{m_{i}} z_{i}^{2}\left(\frac{T_{e}}{\delta}\right) x^{2}}{1+\frac{2}{3}\left(\frac{m_{e}}{m_{i}}\right) z_{i}^{2}\left(\frac{T_{e}}{\delta}\right) x^{2}}\right] T_{i}
$$

where we have introduced

$$
\begin{aligned}
& \delta=\frac{L}{4 R k} m_{e} v_{e i}\left(\frac{8 T_{i}}{\pi m_{i}}\right)^{1 / 2} \\
& x=\left(\frac{3 e \varphi}{2 T_{e}}\right)^{1 / 2}
\end{aligned}
$$

We readily note that if the potential is ignored, the electron and ion escape energies reduce to

$$
E_{L e}=\frac{5}{2} T_{e} ; E_{L i}=2 T_{i}
$$

which agrees with previous results ${ }^{(1)}$. Also note that the calculated quantities $E_{L e}$ and $E_{L i}$ are the average energies of the escaping particles as they leave the plasma chamber. The potential must then be added to the ion energy and subtracted from the electron energy to obtain energies of these particles outside the chamber. Thus the average energy of an escaping electron outside the plasma is $\left(E_{L e}-e \varphi\right)$ whereas that of an escaping ion is $\left(E_{L i}+e \varphi\right)$.

\section{Application to Mars Mission}

We assess the propulsive capability of the GDM fusion thruster by applying it to a Mars Mission. We assume a constant thrust, acceleration/deceleration type of trajectory for which the round trip (RT) time can be written as

$$
\tau_{R T}=\frac{4 d}{g I_{s p}}+4 \sqrt{\frac{d m_{f}}{F}}
$$

where $d$ is the linear distance between Earth and Mars, $m_{f}$ the dry mass of the vehicle, $F$ the thrust, $g$ the Earth's gravitational acceleration and $I_{s p}$ the specific impulse. We choose $d=0.78 \times 10^{11} \mathrm{~m}$, which is the distance between the two planets when they are aligned with the sun, and that occurs approximately every 26 months. The results are shown in Table 1, which also gives the important propulsion and vehicle parameters. In obtaining these results we utilized the virial theorem ${ }^{(4)}$ in calculating the magnet mass using Beryllium-Copper composite for the magnet material and assumed an advanced radiator design and material that allow the rejection of $64 \mathrm{MW}$ per metric ton. 
Table 1. Mars Mission with GDM Propulsion

\begin{tabular}{cccccc}
\hline \hline $\begin{array}{c}\text { Plasma Density } \\
\left(\mathbf{c m}^{-3}\right)\end{array}$ & $\begin{array}{c}\text { Length } \\
(\mathbf{m})\end{array}$ & $\begin{array}{c}\text { Total Mass } \\
(\mathbf{m T})\end{array}$ & $\begin{array}{c}\text { Isp } \\
(\mathbf{s e c})\end{array}$ & $\begin{array}{c}\text { Thrust } \\
(\mathbf{N})\end{array}$ & $\begin{array}{c}\tau_{\mathrm{RT}} \\
(\mathbf{d a y s})\end{array}$ \\
\hline $1 \times 10^{15}$ & 3842 & 11481 & $2.009 \times 10^{5}$ & $1.558 \times 10^{3}$ & 1112 \\
$1 \times 10^{16}$ & 384 & 1373 & $2.000 \times 10^{5}$ & $1.569 \times 10^{4}$ & 123 \\
$2 \times 10^{16}$ & 192 & 970 & $1.998 \times 10^{5}$ & $3.144 \times 10^{4}$ & 74 \\
$3 \times 10^{16}$ & 128 & 947 & $1.996 \times 10^{5}$ & $4.722 \times 10^{4}$ & 60 \\
$4 \times 10^{16}$ & 96 & 1018 & $1.995 \times 10^{5}$ & $6.302 \times 10^{4}$ & 54 \\
$5 \times 10^{16}$ & 77 & 1128 & $1.994 \times 10^{5}$ & $7.882 \times 10^{4}$ & 51 \\
$1 \times 10^{17}$ & 38 & 1848 & $1.991 \times 10^{5}$ & $1.580 \times 10^{5}$ & 46 \\
\hline \hline
\end{tabular}

Although the ion temperature was assumed to be $10 \mathrm{keV}$, they leave the GDM thruster at about $50 \mathrm{keV}$ energy due to the presence of the electrostatic potential whose energy is superimposed on their escape (from the mirror) energy. We note from the above table that increasing the plasma density results in shorter device length and shorter trip time. However, if the mass of the vehicle is the critical factor, then a density of $3 \times 10^{16} \mathrm{~cm}^{-3}$ results in the smallest mass of $947 \mathrm{mT}$ and yields a trip time of about 60 days, which may be more viable economically because of the high cost of putting objects in space. Note that in all instances the specific impulse is approximately the same, i.e. about $2 \times 10^{5}$ seconds, while the thrust varies due to variation in length and density. There was no attempt to optimize the performance of the system due to the many variables that enter the analysis, but some optimization can be carried out, and that will be left to a future effort.

A particularly useful information for any propulsion system is the specific power, which is the ratio of thrust power to mass $(\mathrm{kW} / \mathrm{kg})$, and the specific mass or the thrust to weight ratio, and for the GDM fusion propulsion system these are displayed in Figs. (1) and (2), respectively.

Figure 1. Round Trip Mars Mission with GDM, Isp $=2 \times 10^{5} \mathrm{sec}$




Figure 2. GDM Propulsion System

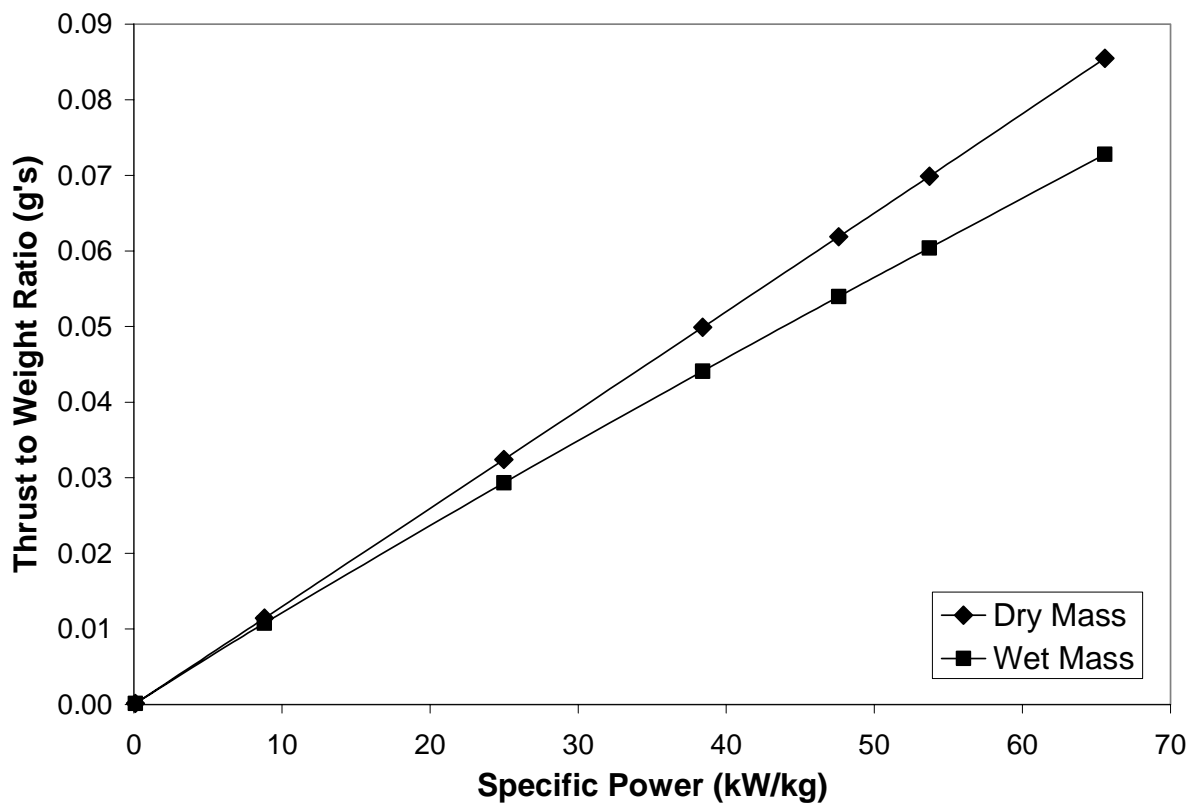

Fig. (1) reveals that the trip time decreases with increasing specific power while Fig. (2) shows that the acceleration increases with increasing specific power. Since the specific power is calculated using the dry mass of the vehicle, the upper curve in Fig. (2) represents the final deceleration while the lower curve represents the initial acceleration. In both cases, it is clear that the acceleration experienced by the GDM propulsion system is quite modest, and that is a consequence of the relatively modest thrust it generates while providing a very large specific impulse. For many interplanetary manned missions the reverse may be more desirable, and in a future publication we will address a GDM design that will yield a large thrust at a more moderate specific impulse.

\section{Acknowledgement}

This work was supported in part by NASA.
References

1. T. Kammash and M.J. Lee, "Gasdynamic Fusion Propulsion System for Space Exploration.” Journal of Propulsion and Power, 11, 544 (1995).

2. D. Rose and M. Clark, Plasma and Controlled Fusion, Wiley, New York (1961), pp. 66.

3. T. Kammash and D.L. Galbraith, "Improved Physics Model for the Gasdynamic Mirror Fusion Propulsion System." Journal of Propulsion and Power, 14, 24 (1998).

4. R.J. Litchford, et al., NASA/TP_2001_211274(2001). 